\title{
Raccontare Zeus. Poesia e cultura di corte ad Alessandria, a partire dall' Inno I di Callimaco
}

\author{
Stefano Giovanni Caneva \\ Università di Pavia
}

\section{Il genere e l'occasione, tra fiction e società di corte.}

Genere, occasione, ricezione: se il nesso che lega un componimento poetico al suo pubblico reale o immaginario costituisce uno dei problemi essenziali di ogni storia letteraria, nel caso della letteratura di corte ellenistica questa tematica ha conosciuto, negli ultimi decenni, una vitalità eccezionale. Le ragioni di tale interesse si riconducono alla necessità di rivalutare la poesia di III secolo alla luce dell'allargamento del patrimonio dei testi conservati ${ }^{1}$, nonché all'urgenza di riscattarla dal giudizio di arte disimpegnata, al quale è stata a lungo relegata. Superata l'interpretazione della poesia di corte come torre d'avorio e gioco dell'arte per l'arte, una maggiore attenzione è stata dedicata alle dinamiche che consentono al testo poetico di entrare in relazione con il suo ambiente di ricezione e di istituire dunque un rapporto specifico con la propria contemporaneità. In questo campo, le ricerche si sono concentrate da un lato sulle strategie discorsive attraverso le quali il testo poetico attiva un processo di comunicazione con il proprio pubblico implicito ${ }^{2}$, dall'altro sul rapporto che il poeta

1 Si pensi al contributo del P.Mil.Vogl. VIII.309 per la conoscenza dell'opera di Posidippo (Austin Bastianini, 2001, e 2002). Per una puntuale bibliografia degli studi recenti, cf. Ambühl, 2007, p. 276-277.

2 Un approccio narratologico ad ampio raggio sulla letteratura ellenistica e le sue premesse arcaiche è offerto da Morrison, 2007. Vamvouri Ruffy, 2004 fornisce un'analisi delle strategie comunicative che coinvolgono narrazione, poeta e ambiente di ricezione negli Inni callimachei, proponendone una sistematica comparazione con gli Inni omerici e con quelli epigrafici. Di particolare interesse per il presente intervento risultano le p. 217-283, dedicate al rapporto fra strategie compositive, erudizione e ideologia di corte ad Alessandria. Sul patronato letterario come sistema di comunicazione impostato sulla triangolazione poeta-patrono-pubblico, si veda anche Ambühl, 2007. Per una lettura dei complessi rapporti fra voce/voci narranti, materia del racconto e destinatari interni nell'opera di Callimaco, cf. anche Goldhill, 1986 e 1991; Bing, 1993; Harder, 1992, 2003 e 2010; Lüddecke, 1998. 
intrattiene con l'ambiente socio-culturale nel quale opera, inquadrando come oggetti di studio la condizione degli intellettuali a corte, le occasioni di performance e comunicazione della cultura e i meccanismi di scambio fra poeti e patroni ${ }^{3}$.

Laddove manchino indicazioni esplicite nelle fonti antiche, la valutazione della dimensione pragmatica della letteratura di corte ha generalmente diviso i critici fra opinioni discordi. L'interpretazione delle marche testuali che, all'interno dei vari componimenti, sembrano rinviare a un contesto di performance reale, sono state talora interpretate come rimandi virtuali, interni alla fiction poetica. Secondo questa lettura, il poeta di corte, pur operando in un contesto in cui la fruizione della letteratura risulta ormai mediata dal testo scritto, attingerebbe a modalità espressive desunte dalla tradizione precedente e dal nesso genere-pubblico che in essa è codificato. Tale prospettiva ha posto l'accento soprattutto sul rapporto dotto, allusivo e spesso non privo di ironia, che il poeta istituisce con i modelli letterari ai quali fa appello, secondo una dialettica che rimanda a un pubblico scelto di lettori, in grado di ricostruire il nesso fra la fiction poetica e la tradizione letteraria di riferimento. Non manca, del resto, un'interpretazione alternativa, secondo la quale alle marche interne al componimento corrisponderebbe una performance reale. Tale lettura attribuisce un peso maggiore alla dimensione socio-culturale dell'occasione di fruizione e ricezione del testo, concentrando l'analisi sulla condizione del poeta all'interno delle dinamiche culturali della corte e sull'efficacia delle relazioni che si instaurano, nell'economia del testo poetico, fra voce narrante, contenuto del racconto e pubblico potenziale ${ }^{4}$.

Tali interpretazioni, pur divergenti, lasciano tuttavia aperte alcune aree di intersezione, sulle quali intendo lavorare a proposito dell'Inno a Zeus di Callimaco. L'alto grado di riflessività con cui la poesia alessandrina affronta il proprio rapporto con i modelli letterari atteggiamento che non esclude, e spesso prevede l'uso distaccato e ironico degli strumenti di genere - non implica infatti che la nuova letteratura si ponga fuori dal circuito comunicativo della performance. Piuttosto, il distacco che il narratore mostra verso la propria materia sembra costituire una scelta di metodo, sulla quale si fonda la possibilità di una riflessione della letteratura sulle proprie risorse e strategie discorsive 5 . Ciononostante, la poesia di corte, che nasce in concomitanza e in sinergia con lo studio filologico e la canonizzazione della tradizione letteraria scritta, se da un lato - secondo l'epistemologia aristotelica che informa la cultura del Museo - mira a diventare a sua volta parte e metro di paragone di quel canone,

3 Per una valutazione storico-sociale del ruolo dell'intellettuale a corte, si vedano Herman, 1997; Weber, 1997; Strootman, 2007, p. 189-250, e Strootman, 2010. Per una analisi del ruolo degli intellettuali nelle differenti corti macedoni, ricordo Weber, 1992 per gli Argeadi; Weber, 1993 e Kerkhecker, 1997 per i Tolemei; Weber, 1995 per gli Antigonidi.

4 Tali posizioni alternative sono espresse rispettivamente da Bing, 1988 e Cameron, 1995. Alla lettura di Bing, per il quale " one might go so far to say that the real world for such a scholar or poet was largely the worlds of books ", si oppone lo storicismo di Cameron, che insiste sul fondamentale ruolo socio-culturale della corte ellenistica come ambiente di promozione e fruizione della letteratura.

5 Come afferma Goldhill, 1991, p. 209, " parody works by making explicit, by drawing (critical) attention to the conventions, strategies and characteristics of its objects. Literary parody conventionally shows how literature requires that its conventions be taken seriously". 
dall'altro passa pur sempre attraverso uno stadio sperimentale, che è quello della presentazione dell'opera d'arte al pubblico della corte. È questo ambiente, infatti, che comprende sia gli intellettuali che vivono nel Museo, sia i sovrani patroni e il loro entourage di philoi e funzionari, a promuovere la poesia alessandrina e a indirizzarne in qualche modo gli sviluppi, con i propri gusti e le proprie preferenze. In questo quadro, in cui la relazione fra autore e pubblico si salda entro gli stretti confini della corte, l'occasione della fruizione diventa un tassello essenziale per l'analisi delle relazioni che intercorrono fra le varie componenti del sistema letterario: la ricerca stilistica del poeta, le esigenze del genere, le aspettative del pubblico e quelle del sovrano mecenate.

Sebbene la poesia alessandrina abbia nella corte il proprio ambiente elettivo, raramente essa rimanda in maniera esplicita alla vita cortigiana, ai suoi personaggi e ai luoghi della sua socialità ${ }^{6}$. La ricostruzione delle dinamiche con cui il testo recepisce ed evoca il proprio ambiente culturale di riferimento appare inoltre tanto più complessa, quanto più stratificate sono le strategie discorsive alle quali generi e autori fanno appello. In questo contesto, la dimensione simposiale con cui si apre l'Inno a Zeus rende necessaria una lettura che si svolga su più livelli, da un lato rintracciando i possibili percorsi intertestuali che collegano il componimento alla tradizione letteraria precedente, dall'altro inserendo le strategie di costruzione del significato in una continua dialettica fra la materia del racconto - la sua fiction letteraria e mitologica - e la cultura della corte alessandrina, nella quale il poeta opera in qualità di intellettuale e philos del sovrano.

Cercherò dunque di focalizzare la mia attenzione sulle strategie discorsive attraverso le quali, nell' Inno a Zeus, Callimaco propone una visione del rapporto fra sovrano e divinità e al contempo delinea il proprio ruolo di intellettuale a corte. Al centro del lavoro sarà l'analisi delle relazioni e delle interferenze fra l'occasione simposiale, le potenzialità e le forzature del genere innodico e le aspettative del pubblico di corte. Cercherò inoltre di mostrare come l'Inno pervenga non solo a offrire, attraverso un complesso percorso di rimandi intertestuali, una certa visione del potere e del suo rapporto con la divinità regale per eccellenza, ma a produrre al contempo una riflessione consapevole sulle risorse del discorso poetico e sulla sua efficacia nel proporre modelli di legittimazione del potere.

\section{L'occasione simposiale dell'Inno a Zeus.}

Attraverso il tradizionale riferimento al dio destinatario del canto, i primi versi dell' Inno a Zeus collocano il componimento nella dimensione di una performance simposiale, che si svolge in occasione di una libagione per Zeus:

Nelle libagioni di Zeus, che altro si potrebbe cantare

di meglio del dio stesso, sempre grande, sempre sovrano? ${ }^{7}$

6 Cf. Kerkhecker, 1997. Tale affermazione è largamente valida, anche se alcuni generi, come l'epigramma (cf. Ambühl, 2007), godono di una specifica libertà nel recepire e rappresentare con immediatezza le occasioni della vita di corte.

7 Call., Hymn. I.1-2. La traduzione italiana dei passi di Callimaco è tratta da D’Alessio, 1996. 
Ateneo ricorda che il simposio è tradizionalmente introdotto da una successione di tre libagioni, la terza delle quali è rivolta a Zeus Soter $^{8}$. Il momento è tradizionalmente dedicato al canto di inni, ma nel contesto delle corti ellenistiche tale occasione accoglie anche i componimenti rivolti al re stesso o a dignitari di corte? ${ }^{9}$ E questo il caso, per esempio, del peana in onore di Seleuco Nicatore, conservato su un'iscrizione di Eritre ${ }^{10}$, come pure dell'Epinicio a Sosibio di Callimaco, nel quale le libagioni sono offerte a Posidone, patrono dei giochi Istmici, dove Sosibio ha riportato il primo premio ${ }^{11}$. Lascerò da parte il problema dell'identificazione del re storico al quale l' Inno sarebbe indirizzato ${ }^{12}$, per concentrarmi sulle indicazioni che il componimento offre in merito alla costruzione dell'immagine della regalità tolemaica nel primo quarto del III secolo a.C.: i suoi attori, il suo ambiente, le sue strategie culturali $^{13}$.

Nell'introdurre il tema del canto, che avrà come oggetto il dio stesso, Callimaco esordisce con un dubbio:

E come lo [Zeus] canteremo, Ditteo o Liceo?

$\grave{E}$ in dubbio il mio cuore, poiché è disputata la nascita ${ }^{14}$.

La domanda riprende il tema dell'incertezza del poeta sul modo migliore di celebrare un dio, ampiamente attestato nella tradizione innodica, focalizzando il problema sulla nascita di

8 Athen. 15.692 F - 693 C.

9 Sul rapporto fra simposio e letteratura in età ellenistica, si vedano Cameron, 1995, p. 71-103, e Murray, 1996, con ulteriori rimandi bibliografici.

10 Coll. Alex. p. 140 Powell, dove il re è rappresentato come figlio di Apollo.

11 Call. fr. 384 Pf.

12 Sono state proposte tre diverse identificazioni del re storico, cui Callimaco farebbe riferimento ai

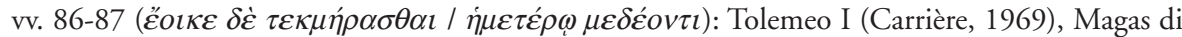
Cirene (Meillier, 1979) e, più frequentemente, Tolemeo II (cf. già Wilamowitz, 1924, II, p. 10-11; più recentemente, cf. Clauss, 1986, cui fanno riferimento vari studi successivi). Basandosi sui dati cronologici raccolti da Koenen, 1977, James Clauss ha proposto (ma si veda già Richter, 1871, p. 1-4) che l'Inno dovesse accompagnare i festeggiamenti tenutisi ad Alessandria, il 12 Dystros 285/4 o 284/3, per il genetliaco e l'ascesa al trono di Tolemeo II come coreggente. Tale festa sarebbe caduta in corrispondenza dei Basileia, festività macedone in onore di Zeus basileus. Che il re cui Callimaco fa riferimento sia un ancor giovane Tolemeo II sembra l'ipotesi più convincente (sugli argomenti per una datazione alta dell' Inno, prima degli anni '70 del III secolo, cf. Clauss, 1986 p. 156-157, n. 5). Ancorché non conclusiva, la ricostruzione proposta da Clauss sottolinea le suggestive corrispondenze fra il tema dell' Inno e la festa per Zeus regale, nonché il rispecchiamento fra il racconto di Callimaco e l'ascesa al trono del Filadelfo, scelto dal Soter a scapito dei fratelli minori (cf. Just. 16.2.7). La stessa finalità del componimento è stata ampiamente discussa e le differenti ipotesi vanno dal vero e proprio inno per una cerimonia religiosa per Zeus, al canto per l'incoronazione del sovrano (per una panoramica sulle varie proposte, cf. Clauss, 1986, p. 159, n. 13). Contro il tentativo di individuare un preciso referente storico del re citato nel poema, si vedano, fra gli altri, McLennan, 1977 e Hopkinson, 1984.

13 Per una analisi dei tratti distintivi della regalità nel primo ellenismo, si vedano, oltre ai classici Moore, 1983, Walbank, 1984 e Samuel, 1993, i più recenti Davies, 2002 e Ma, 2003.

14 Call. Hymn. I.4-5. 


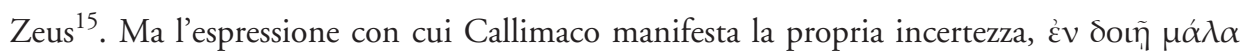

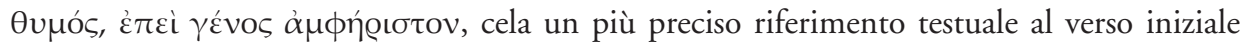

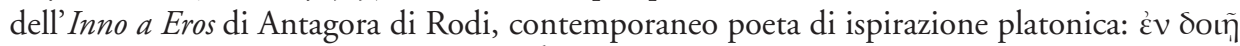

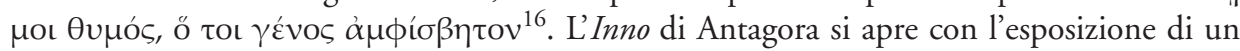
dubbio genealogico sull'origine di Eros, dal quale discende una riflessione sulla duplice natura del dio. In tale duplicità, Martine Cuypers ha proposto di vedere un recupero della distinzione esiodea delle due forme di eris, che nel nuovo contesto innodico attiverebbe una discussione sulla natura dell' eros buono, l'amore filosoficamente teso al raggiungimento del Bene ${ }^{17}$. L'opera di Antagora costituirebbe perciò un'esposizione, in forma poetica, della dottrina platonica dell'eros, per come essa è definita nel Simposio e nel Fedro ${ }^{18}$. Se, come è stato sostenuto, Callimaco è da considerarsi debitore verso Antagora ${ }^{19}$, allora la scelta di questo rimando sembra definire bene, proprio all'inizio dell' Inno, il tipo di contenuto che vi si svilupperà: un discorso simposiale di tenore filosofico, che dalla nascita di Zeus passa a una più ampia discussione sulla natura del dio. Il carattere regale di Zeus, attorno al quale si articola la riflessione sulla legittimazione del potere umano, è del resto sottolineato fin da subito: la tradizionale selezione ed esposizione di miti intorno alla nascita, le imprese e le prerogative del dio, tratto essenziale del genere innodico, è finalizzata a raccontare Zeus in

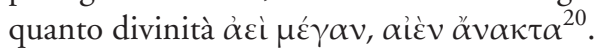

Nel considerare i due possibili luoghi di nascita di Zeus, Creta (identificata prima con il monte Ditte, poi con l'Ida), oppure l'Arcadia, Callimaco inserisce un'affermazione che sembra porre pregiudizialmente fine a ogni dubbio:

\section{Chi dei due, padre, ha mentito?}

"I Cretesi mentono sempre»: anche la tomba tua, Signore,

hanno architettato i Cretesi. Ma tu non sei morto: sei eterno ${ }^{21}$.

La verità non può che stare dalla parte dell'Arcadia, perché $i$ Cretesi mentono sempre. La celebre frase, che la tradizione attribuisce a Epimenide ${ }^{22}$, è la prima delle due citazioni attorno alle quali si articola il complesso percorso argomentativo dell' Inno. La citazione compare qui come parte di un ragionamento che, in modo conciso ma significativo, sposta l'attenzione dalla nascita di Zeus alla sua presunta morte ${ }^{23}$. L'argomentazione si articola attraverso una

15 Si veda in particolare l'Inno a Dioniso (HH XXV.1-7), dove compare anche il rifiuto delle localizzazioni differenti, considerate come menzognere.

16 Coll. Alex., Antag. fr. 1, p. 120 Powell.

17 Cuypers, 2004. Sul legame fra Callimaco e Antagora, cf. anche Stephens, 2003, p. 79-82.

18 Ciò appare coerente con una ben attestata tradizione di III secolo (si pensi a Cleante o ad Arato), intesa a sviluppare la poesia didascalica in chiave filosofica.

19 Su questa successione, cf. Cuypers, 2004, con particolare riferimento all'uso del termine genos nei due passi.

20 Call. Hymn. I.2. Sull'impiego della figura di Zeus nella poesia di corte alessandrina, nel contesto della legittimazione del potere regale, cf. Weber, 1993, p. 355-356, e Hose, 1997.

21 Call. Hymn. I.7-9.

22 Fr. 3 B 1 DK.

23 Per una analisi della sezione dell' Inno dedicata alla nascita del dio, si vedano Stephens, 2003, p. 77-114, e Ambühl, 2005, p. 235-245. 
frase generale, il detto di Epimenide sulla falsità dei Cretesi, cui segue un esempio esplicativo: i Cretesi hanno inventato la tomba di Zeus. L'esempio è subito confutato da un'osservazione di carattere assiomatico: Ma tu non sei morto: sei eterno. Occorre rilevare fin d'ora un punto sul quale si tornerà in seguito: la frase di Epimenide costituisce un famoso paradosso logico, essendo Epimenide stesso cretese, e dunque mentitore. La debolezza dell'argomentazione, e l'assenza di paralleli noti per la nascita arcade di Zeus, sembrerebbero pertanto suggerire una costruzione dell'intero passo in chiave ironica, e hanno infatti indotto vari interpreti a privilegiare una analisi esclusivamente letteraria dell' Inno, marginalizzando l'interpretazione dei referenti culturali esterni al testo ${ }^{24}$.

Tale lettura sembra però lasciare in secondo piano l'importante riferimento alla tomba cretese di Zeus, sulla cui falsità Callimaco si esprime anche altrove, nel frammento 202 Pf.:

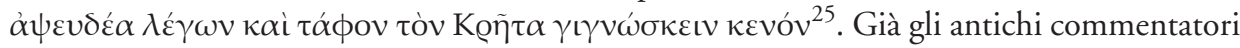
vedevano nella tomba di Zeus un rimando all'opera di Evemero, alla quale, del resto, il I Giambo callimacheo riserva una secca bocciatura. Attraverso la voce del redivivo poeta Ipponatte, Callimaco bolla infatti l'autore di Messene come falsario e scrittore di adika biblia $^{26}$. Tale critica sembra superare i confini della semplice polemica stilistica, nella misura in cui individua la colpa dell'autore nel cuore concettuale della Hiera Anagraphe, l'invenzione dello Zeus di Panchaia. Nel paragrafo successivo si cercherà pertanto di leggere l'Inno a Zeus sullo sfondo dell'opera di Evemero, nel tentativo di fare emergere i possibili legami che il testo callimacheo istituisce con la cultura del suo tempo.

\section{La Hiera Anagraphe e l'Evemerismo.}

La Hiera Anagraphe ci è nota attraverso frammenti di tradizione indiretta, in particolare la selezione fatta da Diodoro nel V e VI libro della Biblioteca e le citazioni che, nel I libro delle Divinae Institutiones, Lattanzio deriva dalla versione latina di Ennio ${ }^{27}$. La lettura delle testimonianze offre un'immagine dell'opera di Evemero che ben si armonizza con una tradizione di lunga data, intesa a costruire una storia della civiltà attraverso una rilettura

24 Tra questi, si veda Lüddecke, 1998.

25 Fr. 202.15-16 Pf. Per una panoramica dei passi antichi inerenti l'esistenza di una tomba cretese di Zeus, cf. Kokolakis, 1995. Sul legame Zeus - Creta, cf. anche Bremmer, 2009.

26 Fr. 191.9-11 Pf.: Qui tutti al tempio davanti alle mura venite, dove il falsario dell'antico Zeus di

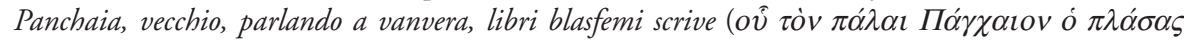

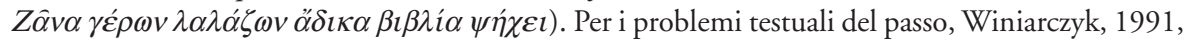
p. 1. Sulla polemica letteraria nel I Giambo, cf. Kerkhecker, 1999, p. 11-48 (su Evemero, p. 22-25); cf. Lelli, 2004. La priorità di Evemero rispetto all'Inno a Zeus, seppur non dimostrabile in modo conclusivo sulla base dei rimandi presenti nelle opere callimachee, è sostenuta già dai commentatori antichi e generalmente accettata dagli studiosi moderni. Per una rassegna dei passi antichi che attribuiscono a Callimaco posizioni anti-evemeristiche, cf. Fraser, 1972, p. 289-296; Kokolakis, 1995, 128-129; Winiarczyk, 2002, p. 3-5 (anche sulle varie ipotesi di datazione della Hiera Anagraphe).

27 I frammenti di Evemero sono raccolti in FGrH 63; Vallauri, 1956; più recentemente, Winiarczyk, 1991. Una recente monografia sull'autore è stata pubblicata da Winiarczyk, 2002. 
del mito che potremmo definire, in termini generali, razionalizzante e allegorizzante ${ }^{28}$. A questo scopo, Evemero impiega strumenti narrativi come l'eziologia, la paraetimologia, la ricerca del protos heuretes, rileggendo e reinventando il mito e individuandovi le tracce per la costruzione di un passato pre-storico dell'umanità, sub specie mythologiae. La novità di Evemero sta però nell'inserire tali strategie espositive nella cornice del racconto di un viaggio straordinario in un arcipelago situato al largo dell'Oceano, a oriente dell'Arabia. Il racconto delle origini è pertanto adattato al genere narrativo della paradossografia, e più precisamente al filone del resoconto di lontane società utopiche, di grande successo nell'epoca successiva ad Alessandro.

Il racconto che Evemero offre su questo passato primitivo prende l'avvio proprio dalla descrizione della più significativa fra le meraviglie osservate dal viaggiatore sull'isola di Panchaia: il tempio di Zeus Triphylios. L'antichissimo santuario, del quale sono descritte la struttura, le decorazioni e le principali pratiche cultuali, contiene al suo interno delle iscrizioni che i sacerdoti fanno risalire al periodo della sua edificazione, voluta da un antichissimo re chiamato Zeus, cui il tempio è dedicato. All'interno del tempio, poi, sorge una grande stele d'oro, sulla quale lo stesso re ha inciso in caratteri geroglifici le gesta sue e del nonno Urano, e Hermes ha successivamente integrato le informazioni relative ai suoi successori, Artemide e Apollo ${ }^{29}$. Il contenuto dell'iscrizione sacra traspone le genealogie divine, contenute nelle teogonie tradizionali, sul piano della storia umana, in una ricostruzione che si pretende cronologicamente universale, perché estesa a tutto il mondo e risalente fino alle origini dell'umanità. Coerentemente, le lotte teogoniche sono evocate nella forma di vicende dinastiche che riguardano un'unica famiglia con le sue varie diramazioni, quella dei discendenti del primo re, Urano: Zeus è l'erede che, nascosto alla nascita per sfuggire alle trame dei parenti, torna per rivendicare il proprio diritto al trono, combatte guerre contro i propri rivali e infine diviene signore del mondo, unendo le prerogative regali di conquistatore, detentore della giustizia e benefattore, che accoglie e incentiva le scoperte e le invenzioni utili per migliorare la vita dell'umanità ${ }^{30}$.

Ma un altro punto risulta centrale per il presente discorso: durante il suo regno, Zeus procede alla realizzazione di un progetto di consolidamento del potere attraverso l'istituzione di culti in onore suo e della propria famiglia. Così Zeus non solo edifica il tempio di Panchaia, ma fonda anche un culto in nome del nonno Urano, re e primo astronomo, il cui nome è trasferito al cielo stesso. Inoltre, nel corso dei suoi viaggi, Zeus stringe alleanza con i re locali,

28 Sulla tradizione della Kultursgeschichte greca dal V secolo (con particolare attenzione al ruolo di Democrito) alle sue evoluzioni ellenistiche e romane, si veda il classico Cole, 1967; cf. Bremmer, 2007. Sulle posizioni di Prodico in relazione agli sviluppi ellenistici della teoria della divinizzazione dei benefattori, cf. Henrichs, 1984. Per una ricostruzione dell'opera di Ecateo di Abdera rimando anche a Murray, 1970; Fraser, 1972, p. 496-498; Dillery, 1998. Su Evemero di Messene, cf. anche Fraser, 1972, p. 289-296, Müller, 1993 e Stephens, 2003, a proposito dell'assorbimento di elementi egizi nella tradizione greca. In generale, sull'allegoria come strumento di reinterpretazione e di riattualizzazione del mito, si veda Brisson, 1996.

29 Diod. V.46, 7 (= 37 Winiarczyk).

30 Lactant. Div. Inst. I.11, 35; 13, 2 (= 67, 66 Winiarczyk). 
e il rapporto di amicizia è sancito dalla fondazione di templi e feste annuali in suo onore, secondo un modello ricalcato sull'operato dei re ellenistici contemporanei di Evemero ${ }^{31}$.

Evemero ribadisce continuamente il legame fra il suo Zeus mortale e Creta. Non solo, infatti, Zeus combatte contro lo zio Titano a capo di un esercito di Cretesi, e torna all'isola dopo aver liberato dalla prigionia il padre Crono ${ }^{32}$, ma, dopo essere divenuto a sua volta re, porta con sé a Panchaia una gruppo di suoi fedeli provenienti da Creta, alla cui discendenza sarà riservato il compito di servire come sacerdoti del tempio di Zeus Triphylios ${ }^{33}$. Infine, dopo aver regnato sul mondo intero, Zeus si ritira a Creta, dove la sua sepoltura, secondo il passo di Lattanzio, è collocata presso la città di Cnosso, e reca l'epitafio ZAN KPONO؟ ${ }^{34}$.

Un'interpretazione della Hiera Anagraphe non può prescindere dall'analisi dei suoi rapporti con la realtà politica e culturale del suo tempo. La lettura della storia dell'umanità attraverso il filo rosso delle vicende di una casata regnante è figlia della nuova realtà politica del Mediterraneo dopo Alessandro, dove il bios del sovrano e la sua genealogia divengono il filo rosso dell'esposizione storica, così come della giustificazione del potere da parte dei Diadochi, che al Macedone e alla sua famiglia fanno appello per fondare la propria legittimità. Ma nell'opera di Evemero c'è soprattutto un tema che occorre leggere alla luce della realtà politica e culturale del periodo successivo ad Alessandro: la riflessione sulla diffusione dei culti regali. Proprio in questa prospettiva si coglie l'aggiornamento, al panorama politico e religioso del III secolo, della tradizione razionalizzante ed allegorizzante del mito cui si è accennato, e in particolare del ricorso alla teoria della divinizzazione di uomini benefattori per scrivere una storia del progresso della civiltà umana.

Tale filone interpretativo trova significativi antecedenti nella cultura sofistica di $\mathrm{V}$ secolo. La nascita della religione era ricondotta da Prodico di Ceo a una tappa dell'evoluzione culturale dell'uomo: gli antichi uomini avrebbero riconosciuto uno statuto divino dapprima alle potenze naturali che risultavano per loro più utili, quindi a uomini distintisi per la scoperta di tecniche e istituzioni tali da fare progredire le condizioni della vita umana ${ }^{35}$. Sul piano dello sviluppo del potere, Crizia interpretava l'istituzione del culto degli dei come inganno politico, atto a sancire l'ordine del più forte ${ }^{36}$. La lettura sofistica storicizzava e umanizzava il mito, secondo una prospettiva destinata a divenire asse portante per il superamento della distinzione fra tempo degli dei e tempo degli uomini ${ }^{37}$. Ma la svolta che permette di giungere alla maturazione dell'Evemerismo arriva solo in epoca ellenistica, allorché la teoria della

31 Per il culto di Crono, Lactant. Div. Inst. I.11, 63. Sull'istituzione di culti personali da parte di Zeus, Lactant. Div. Inst. I.22, 21-27 (= 64A Winiarczyk).

32 Lactant. Div. Inst. I.14, 10-12 (= 56 Winiarczyk).

33 Diod. V.46.3 (= 35 Winarczyk), dove si afferma che le prove di questa discendenza sono di carattere linguistico: i sacerdoti di Panchaia conservano ancora alcune espressioni del dialetto cretese.

34 Lactant. Div. Inst. I.11, 44-48 (= 69A Winiarczyk).

3584 B 5 DK.

3688 B 25,1-2 DK.

37 Esempi significatici sono offerti dalla tradizione attidografica, nella quale Dioniso e Demetra sono considerati come inventori itineranti, il cui arrivo in Attica è datato e collocato ai tempi dei primi re della regione: cf. Marmor Parium, FGrH 239 A 12-13 (Per altri esempi, Henrichs, 1984, p. 144145). 
divinizzazione di antichi benefattori è adattata agli sviluppi contemporanei delle strategie di legittimazione del potere, e in particolare all'istituzione dei culti regali.

È ciò che accade, ancor prima che nella Hiera Anagraphe, negli Aigyptiaka di Ecateo di Abdera, opera scritta sul finire del IV secolo alla corte di Tolemeo I, di cui ampi stralci sono contenuti nel I libro della Biblioteca di Diodoro ${ }^{38}$. I passi diodorei attribuibili a Ecateo di Abdera offrono un esempio di come l'indagine etnografica condotta presso la nuova corte ellenistica potesse procedere di pari passo con la giustificazione della regalità, messa in atto dalla corte stessa. La fonte diodorea interpreta l'antichità pre-dinastica egizia alla luce della teoria sulla distinzione fra divinità celesti e divinità di origine umana: la tradizione egizia per la quale gli dei governarono l'Egitto fino a Horus, prima dell'avvento delle dinastie umane ${ }^{39}$, è così tradotta nel racconto secondo il quale alcune divinità, e Osiride in particolare, furono in realtà antichissimi re mortali, assunti fra gli dei in virtù della gratitudine dell'umanità verso la loro energesia ${ }^{40}$.

$\grave{E}$ in questo contesto che occorre considerare le peculiarità del pensiero di Evemero. Anzitutto, Prodico - ripreso nel I libro di Diodoro - individuava due fasi nella costituzione del pantheon divino, il culto di potenze divine celesti e quindi quello di uomini divinizzati a causa delle loro opere, delle quali Evemero sembra privilegiare la seconda. Per quanto la brevità dei frammenti non permetta di trarre conclusioni certe, ha forse ragione Thomas Cole ad affermare che la distinzione fra dei celesti e uomini divinizzati è superata in Evemero a favore di questi ultimi: nell'istituire il culto di Urano, il cielo, Zeus introduce di fatto un culto dinastico rivolto a un mortale, il fondatore della propria casata. Inoltre, nella Hiera Anagraphe sembra passare in secondo piano la ragione che in Prodico ed Ecateo appariva fondamentale per la divinizzazione di un mortale: l'euergesia verso l'umanità. Se è vero, infatti, che lo Zeus di Evemero si presenta come patrono e punto di riferimento per gli inventori del suo tempo, risulta altrettanto chiaro che il suo culto non è istituito per via della pietà popolare, ma in riferimento a un progetto volontario di consolidamento del potere. $\grave{E}$ appunto in questi termini che Sesto Empirico si esprime a proposito di Evemero:

Evemero, il cosiddetto ateo, dice che, quando la vita degli uomini era priva di un ordine, coloro che si distinguevano per forza e intelligenza, in modo tale che tutti vivevano sottostando ai loro comandamenti, si diedero da fare per ottenere maggiore ammirazione e maestà, e plasmarono intorno a sé una qualche straordinaria potenza divina, in virtù della quale furono ritenuti dei anche presso i piür1.

38 Sulla possibilità di attribuire ampie sezioni del I libro di Diodoro a Ecateo, cf. Murray, 1970, Fraser, 1972, p. 496-498 e Dillery, 1998. Per una posizione scettica, si vedano Spoerri, 1959 e, da ultimo, Winiarczyk, 2002, p. 11-29.

39 Facendo appello ai resoconti dei sacerdoti egiziani, la fonte diodorea offre una scansione cronologica della storia d'Egitto che annovera diciottomila anni di regno di dei ed eroi, seguiti, dopo Horus, da sovrani umani (Diod. I.44.1). Sui problemi inerenti la cronologia egiziana in Diodoro e nella sua fonte, Ecateo, cf. Murray 1970, p. 145, 149. Sul regno degli dei nella tradizione egiziana, Grimal, $2002^{4}$, p. $56-59$.

40 Diod. I.13.

41 Sext. Empir. Adv. mathem. IX 17 (= 27 Winiarczyk). Trad. it. dell'autore. 


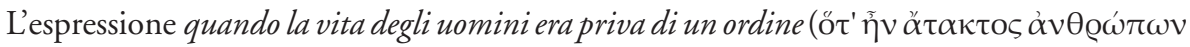
ßíos), con cui esordisce il passo, riprende il passo del Sisifo di Crizia, in cui l'origine della religione è inquadrata come inganno politico inteso a mettere fine al disordine naturale e a imporre l'ordine dei più forti ${ }^{42}$. Sollevando il velo del discorso evergetico e illuminando i rapporti di forza che gli sono sottesi, la Hiera Anagraphe sembra pertanto aprire uno squarcio sul dibattito intellettuale che dovette accompagnare il radicamento dei culti regali, ma che così poco traspare dalla documentazione in nostro possesso, quasi sempre condizionata da occasioni comunicative che impongono temi e linguaggi di ordine politico-diplomatico o religioso ${ }^{43}$. In Evemero, la divinizzazione dei re è spogliata della sua patina idealizzante, nella misura in cui l'attenzione si distoglie dalle divinità celesti per concentrarsi invece sull'autopromozione degli antichi re umani al rango divino. Ma umanizzando il divino ${ }^{44}$, Evemero svela la natura tutta umana del potere. Come afferma Thomas Cole, con Evemero "the Hellenistic monarch ... is now installed in the seat of Zeus himself " ${ }^{45}$. Proprio nello svelamento di questa realtà sembra risiedere l'empietà politica della Hiera Anagraphe. Quale giustificazione del potere regale può infatti essere conservata, se Zeus stesso, dio sovrano per eccellenza, è privato della divinità, da cui promana la sua autorità sacra?

\section{Callimaco e il legame fra Zeus e il re.}

Sta qui, a mio avviso, la chiave di lettura della rivendicata eternità di Zeus nell'inno callimacheo. Accingendosi a dipingere i tratti del supremo dio regale, Callimaco deve innanzitutto rendergli la divinità, che sola può fare di Zeus il modello e la fonte di autorità per il perfetto re umano. La prodigiosa nascita di Zeus e la sua ascesa al trono degli dei sono perciò le risorse che l'autore ha a disposizione per definire, su base mitologica, i fondamenti del potere regale. Da questo punto di vista, l'affermazione della morte di Zeus assume i toni della maldicenza, della proclamazione empia che occorre negare per ricondurre il rapporto fra re e divinità a un modello diverso da quello evemeristico: anziché trasformare gli dei in antichi re umani, occorrerà affermare il carattere divino della regalità contemporanea, che da Zeus promana al re mortale, eletto dal dio. Tale processo non implica però una negazione a priori

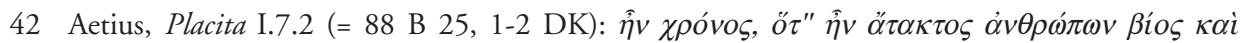

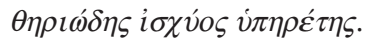

43 Nel campo delle concrete relazioni fra il re e le poleis, l'egemonia del sovrano sulla città o sulla federazione è espressa nei termini paternalistici del re salvatore e benefattore, garante della libertà dei Greci, al quale sono tributati onori eroici o divini, riconducibili nell'alveo dei riconoscimenti attribuiti tradizionalmente dalle città a ecisti, guerrieri, legislatori e altri benefattori della comunità. Sui culti regali del primo ellenismo è oggi disponibile un'ampia bibliografia specialistica. In generale, oltre al classico Habicht $1970^{2}$, si veda il recente Chaniotis, 2003. Sulle premesse di V secolo, cf. Mari, 2004. In relazione alle singole dinastie: sui Tolemei, Pfeiffer, 2008; sui Seleucidi, $\mathrm{Ma}, 2002^{2}$ (con un'ottima trattazione generale delle problematiche inerenti il nesso fra culti regali e discorso della legittimazione), Van Nuffelen, 2004, Chaniotis, 2007; sugli Antigonidi, Mari 2008.

44 Si vedano al proposito le parole di Plut. De Is. 23 p. 360 A,7 (= 15 Winiarczyk): $\dot{\varepsilon} \xi \alpha \nu \theta \rho \omega \pi i \zeta o v \tau \imath$ $\tau \dot{\alpha} \theta \varepsilon \hat{\imath} \alpha$.

45 Cole, 1967, p. 162. 
del valore dei culti per la famiglia regale, che anzi Callimaco sostiene nei componimenti in cui il riferimento all' entourage tolemaico è più apertamente esplicitato ${ }^{46}$. Piuttosto, il rifiuto degli sviluppi teorici dell'evemerismo fissa il dibattito su un piano intellettuale, che concerne la definizione delle risorse dalle quali il discorso di legittimazione del potere deve trarre le sue basi.

Una nuova citazione letteraria, la frase esiodea per cui $i$ re vengono da Zeus, introduce la pars construens di tale riflessione ${ }^{47}$. Lo stretto legame fra il dio regale e il sovrano terreno sta alla base di una serie di corrispondenze fra le prerogative di Zeus e quelle dei re. Il dio trova nel sovrano il corrispettivo del proprio grado fra i mortali, secondo una gerarchia parallela che associa uomini di minor spicco a divinità via via minori. I diversi compiti del re si rispecchiano pertanto in quelli di Zeus: il re custodisce le città, così come Zeus è insediato a protezione delle loro rocche (vv. 81-82); Zeus, legislatore dei Celesti (v. 3), sovrintende al compito regale di gestire la giustizia, distinguendo a questo proposito fra buoni e cattivi re

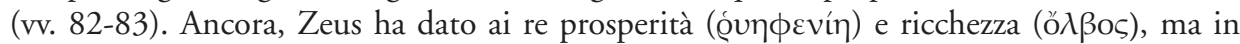
misura diversa: il successo del sovrano è indizio dell'appoggio di cui egli gode presso il dio (vv. 84-86). Un'altra prerogativa del re, la rapidità con cui passa della progettazione (vońon) alla realizzazione $(\tau \varepsilon \lambda \varepsilon \tilde{\imath})$ dei suoi grandi piani (vv. 87-90), trova riscontro nella rapidità con cui

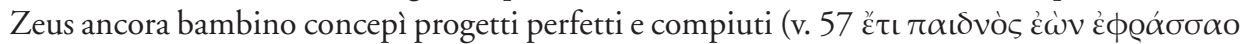

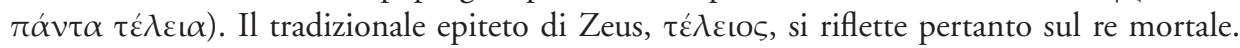

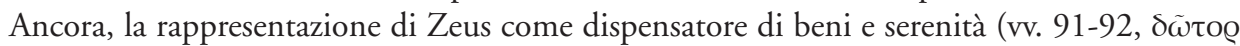
$\dot{\varepsilon} \alpha \omega \omega v, \delta \tilde{\omega} \tau o \varrho \dot{\alpha} \pi \eta \mu o v i ́ \eta \varsigma)$ corrisponde all'immagine del re come garante della prosperità e della sicurezza del suo regno. La funzione di Zeus come fonte di giustizia e prosperità è inoltre evocata, in forma allegorica, attraverso i riferimenti ai personaggi della nutrice Adrastea e della capra Amaltea (vv. 47-49): Adrastea è, come suggerisce il nome, "Colei cui non si sfugge", cioè, tradizionalmente, la giustizia come ordine infallibile di Zeus ${ }^{48}$; il corno di Amaltea è invece la cornucopia, ampiamente utilizzata nell'iconografia regale dell'epoca come simbolo della prosperità garantita dalla coppia dei sovrani ${ }^{49}$. Infine, il commiato dell'inno (vv. 94-96),

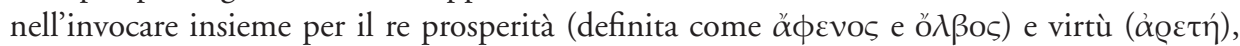
completa l'indicazione relativa al fondamentale rapporto fra Zeus e il sovrano: se il benessere e i successi del re sono i segni della protezione del dio, la rettitudine morale è il dono che giustifica il potere del re mortale, e ne fa l'incarnazione del sovrano perfetto.

Ma il filo argomentativo dell' Inno passa per un ulteriore snodo cruciale. Sostenendo che il dominio sul cielo fu attribuito a Zeus con il consenso dei fratelli maggiori, Callimaco ricorda

46 Sulla posizione di Callimaco nell'ambito del rapporto fra poesia di corte e culti regali, cf. Weber, 1993, p. 243-276.

47 Theog. 96, ripreso in H.H. XXV.4. Sulle riprese esiodee in Callimaco, cf. Reinsch-Werner, 1976 (in particolare p. 24-73 per l'Inno a Zeus).

$48 \mathrm{Si}$ tratta di un tema comune nella poesia di corte alessandrina. Apollonio Rodio lo sviluppa attraverso un'allusione astronomica: il gioco che Adrastea confezionò per Zeus bambino è una sfera composta di cerchi rotanti, che riproduce l'universo (Arg. III.132-141). Allegoricamente, il gioco di Adrastea prepara pertanto il dio bambino al mantenimento dell'ordine cosmico, compito che eserciterà dopo la sua ascesa al potere (cf. Caneva , 2007, p. 82).

49 Al riguardo, cf. Thompson, 1973; Rice, 1983, p. 202-208; Müller, 2009, p. 203-206. 
e smentisce la tradizione secondo la quale i Cronidi avrebbero diviso a sorte le rispettive sedi. Tuttavia, dopo aver sconfessato gli antichi poeti che sostennero questa versione, Callimaco si augura di essere anch'egli mentitore, purché convincente ${ }^{50}$. La versione dell'estrazione è attestata in Omero ( $I l$. XV.187 ss.) e in Pindaro ( $O l$. VII.54 ss.), ma la possibilità di rettificare una tradizione mitica precedente, attraverso l'irruzione della voce narrante nel racconto, è una modalità espressiva che Callimaco deriva dallo stesso Pindaro, presso il quale la libertà dell'autore nel selezionare o rifiutare specifici tratti della tradizione letteraria precedente risulta strettamente legata al fine celebrativo del componimento, e rimanda alle dinamiche intercorrenti fra autore e committente/destinatario. Il richiamo alla possibilità della poesia di rendere credibile ciò che è fiction attiva perciò, nel testo callimacheo, una complessa sottotraccia, che presuppone le precedenti riflessioni sul rapporto fra verità e finzione letteraria nella tradizione poetica greca ${ }^{51}$. La chiave interpretativa della selezione del mito nell' Inno a Zeus sembra dunque individuabile in una forzatura del genere innodico a favore di quei criteri pragmatici che, nella poesia encomiastica pindarica, regolano la scelta della materia in funzione della celebrazione del committente/destinatario.

Centrale, in questo contesto, appare il nesso che il poeta instaura fra la selezione della materia mitica e i criteri di valutazione della propria veridicità. È stato notato ${ }^{52}$ che, in Pindaro, l'efficacia dell'elogio poetico dipende dall'affidabilità di chi lo compone, ma il vaglio secondo i criteri della veridicità riguarda soltanto le informazioni esposte a proposito del personaggio reale da celebrare. Questo è solo uno dei livelli sui quali il poeta encomiastico opera, e nemmeno il più significativo. L'intervento dell'autore si svela invece appieno nella selezione dell'exemplum mitico, che è chiamato a trasfigurare la vicenda umana del patrono su un piano eroico. A questo livello, il criterio di valutazione del valore del poeta non sta nella verosimiglianza della materia mitica selezionata, bensì nella sua pertinenza, ovvero nella sua capacità di «sollecitare la riflessione intorno a temi e problematiche attuali racchiusi nel paradigma eroico e delucidati da esso $"$ " 3 .

Il mito si configura così come un vasto patrimonio di fiction, entro il quale il poeta seleziona ciò che più si adatta al messaggio che vuole comunicare al pubblico, patrono compreso. Questa operazione implica talora una presa di distanze da versioni alternative, attestate nella tradizione letteraria precedente ${ }^{54}$. Se, in età ellenistica, la catalogazione mitografica non fa che accrescere la consapevolezza della contraddittorietà e mendacità di tanti particolari del mito, nondimeno il racconto tradizionale conserva una sua utilità, nella misura in cui contribuisce

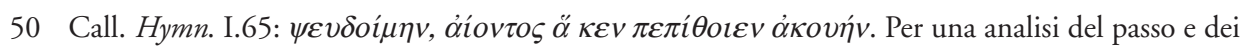
suoi legami con la pratica poetica pindarica, si vedano Fuhrer, 1988; Lombardi, 1998; Floridi, 2004; Morrison, 2007, p. 120-123.

51 Si pensi già a Hes. Theog. 27-28. Sulla fizionalità nella tradizione poetica greca, si vedano anche, oltre alla bibliografia citata in n. 50, Rösler, 1980; Pratt, 1993; Finkelberg, 1998.

52 Per questa lettura, cf. in particolare Floridi, 2004.

53 Floridi, 2004, p. 68.

54 Sull'autonomia del narratore callimacheo nell' Inno a Zeus, cf. Morrison, 2007, p. 115-123. 
a dare credibilità al discorso in cui è inserito ${ }^{55}$. Poiché la scrittura poetica poggia per sua natura su un racconto che recepisce e genera a sua volta nuova fiction ${ }^{56}$, l'augurio esposto da Callimaco al v. 65 tocca l'essenza stessa dell'opera del poeta, che consiste nel selezionare la materia mitologica in funzione del proprio obiettivo comunicativo. Letto in questa chiave, anche il paradossale sillogismo costruito attorno alla citazione di Epimenide si rivela utile al proposito del poeta: pur appoggiandosi su premesse logiche labili, esso serve a privare di autorità il racconto cretese sulla tomba di Zeus e a introdurre quello della nascita prodigiosa e dell'immortalità del dio regale.

\section{Poesia, corte e regalità}

Se l'autorità del re umano è collegata a Zeus grazie alla citazione di Esiodo, è ancora alla Teogonia che Callimaco rimanda per configurare il ruolo del poeta come protetto di Apollo ${ }^{57}$. La definizione del rapporto fra re e poeta che ne deriva, si allontana molto dal modello esposto da Teocrito nell' Encomio di Tolemeo ${ }^{58}$. Allo scambio fra il patrono e il poeta che gli dona fama immortale si sostituisce, in Callimaco, un messaggio fondato sulle relazioni spaziali interne al testo. Il poeta, protetto di Apollo, si colloca al livello che immediatamente precede il re, posto a sua volta al culmine della gerarchia umana, come corrispettivo terreno di Zeus. Al poeta non è assegnato il compito di celebrare a posteriori la grandezza del patrono, ma di illuminare il legame che esiste fra il re e il dio ${ }^{59}$. In altri termini, se la selezione della materia mitica rimanda al nesso poeta-committente, tipico della poesia encomiastica, le strategie espressive dell'inno garantiscono che la figura del patrono terreno si perda in quella del dio regale, che gli dà autorità. Il parallelismo fra Zeus e Tolemeo, che apre l'elogio di Teocrito, lascia il posto, in Callimaco, a un continuo scarto fra il destinatario interno dell'inno, Zeus, e il suo referente esterno, il sovrano patrono. Parallelamente, il riferimento letterario a Esiodo offre lo strumento per suggerire, all'interno del testo poetico, un modello reale di rapporto fra il basileus e il poeta, che ne è philos e consigliere.

La parola poetica si affida pertanto alle risorse espressive e argomentative che le sono proprie, per proporsi come elaboratrice di modelli culturali che agiscono sulla realtà: raccontare Zeus significa trasfigurare la discussione sulla regalità legittima, collocandola su di un piano metastorico, la cui ambientazione è offerta dai temi e dai simboli del mito allegorizzato. Ma l'importanza di tale compito è tanto grande, da non poter essere concessa a tutti. L'inizio dell' Inno, con il riferimento alle libagioni che aprono il simposio, e l'immagine finale del rapporto fra poeta e re, sembrano infatti suggerire precisi limiti spaziali, oltre i quali il lavoro sul mito del dio regale cessa di essere legittimo. La poesia definisce uno spazio

55 Sul mito come fiction, utile nella misura in cui serve ad accrescere la pietà e il senso di giustizia degli uomini, si vedano Strabo 1.2.8-9 e Diod. 1.2.2 (cf. Floridi, 2004, p. 70). Sul lavoro mitografico in età ellenistica, Pellizer, 1993; per le sue influenze sull'Inno a Zeus, Lombardi, 1988.

56 Cf. McLennan, 1977; Floridi, 2004, p. 70.

57 Call. Hymn. I. 78; cf. Hes. Theog. 93-97.

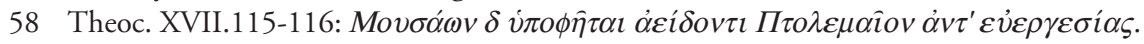

59 Il fatto che da tale legame discenda la legittimità del re costituisce un ulteriore rimando alla Teogonia (Hes. Theog. 93-96). 
simbolico, gerarchico e delimitato, e tale spazio trova il proprio corrispettivo reale nella corte $^{60}$. Che tale spazio possa essere pensato non semplicemente come luogo immaginario, ma collegato alla vita sociale della corte, non è del resto in contrasto con il fatto che, in età ellenistica, la trasmissione della letteratura procede ormai definitivamente per via scritta. Piuttosto, la prospettiva di un'occasione di performance aggiunge sostanza al complesso lavoro di costruzione del significato che l'Inno mette in atto, chiamando in causa riferimenti letterari e culturali noti al suo pubblico.

La vita culturale della corte prevede numerose occasioni di socialità, dalla coabitazione dei sapienti nel Museo alle feste per il grande pubblico, il popolo dei sudditi, dei mercanti e dei diplomatici presenti nella capitale. La complessità del discorso sviluppato da Callimaco sembra suggerire, per il pubblico dell'Inno, una dimensione intermedia, che ben si armonizza con l'immagine del simposio, evocata dal componimento stesso. I fruitori dell' Inno a Zeus potrebbero essere individuati in quel pubblico selezionato e colto che ha accesso al banchetto del re, occasione che in età ellenistica è ancora dedicata - come ha mostrato Alan Cameron ${ }^{61}$ - alle perfomance e alle discussioni dotte. Dall'Inno a Zeus sembra dunque emergere un ambiente, nel quale la riflessione sulla regalità, pur non venendo meno alla celebrazione del potente patrono, può svilupparsi attraverso un discorso poetico complesso, che spinge alla massima intensità il dialogo fra il poeta e la tradizione letteraria precedente. In questo contesto, la libertà nell'uso consapevole e talvolta ironico degli strumenti espressivi non compromette l'efficacia del messaggio poetico, anzi sembra proporre proprio nella poesia e nelle sue complesse dinamiche semantiche la più valida risorsa per la costruzione di un discorso sulla legittimazione del potere regale.

\section{Bibliografia}

АмвÜHL, A., 2005, Kinder und Junge Helden. Innovative Aspekte des Umgangs mit der literarischen Tradition bei Kallimachos, Leuven.

АмвÜнt, A., 2007, Tell, All Ye Singers, My Fame. Kings, Queens and Nobility in Epigram, in P. Bing, J.S. Bruss (eds), The Brill's Companion to Hellenistic Epigram, Leiden - Boston, p. 275-294.

Austin, C., Gallazzi, C. Bastianini, G., 2001, Posidippo di Pella-Epigrammi (P. Mil. Vogl. VIII 309), Papiri dell' Università degli Studi di Milano VIII, (Editio maior), Milano.

Austin, C., Bastianini, G., 2002, Posidippi Pellaei quae supersunt omnia, (Editio minor), Milano.

$60 \mathrm{Al}$ contrario, lo Zeus umano di Evemero, intento a percorrere il mondo e a fondare i propri culti, e per giunta evocato nel resoconto paradossografico di un viaggio fittizio, si colloca fuori dallo spazio elettivo della corte e dalle sue dinamiche ideologiche. Sarà forse proprio questa lettura spaziale, fondata sul rapporto di inclusione/esclusione rispetto alla corte, ai suoi valori e alle sue occasioni di socialità, a offrire una chiave interpretativa al riferimento sprezzante che il redivivo Ipponatte riserva a Evemero nel I Giambo? Qui l'autore di Messene è rappresentato come un vecchio intento a comporre libri blasfemi, lontano dalla corte, presso il tempio di Serapide. Sui problemi inerenti la scelta del luogo da parte di Callimaco e la sua identificazione nella topografia alessandrina, cf. Kerkhecker 1999, p. 22-23; Winiarczyk, 2002, p. 4, n. 14.

61 Cameron, 1995, p. 24-103. 
BIng, P., 1988, The Well-Read Muse. Present and Past in Callimachus and the Hellenistic Poets, Göttingen.

BING, P., 1993, Impersonation of Voice in Callimachus' Hymn to Apollo, TAPhA, 123, p. 181-198.

Bremmer, J. N., 2007, Atheismus in Antiquity, in M. Martin (ed.), The Cambridge Companion to Atheismus, Cambridge, p. 11-26.

Bremmer, J. N., 2009, Zeus' Own Country: Cult and Myth in the Pride of Halicarnassus, in U. Dill, Ch. Walde (Hg.), Antike Mythen. Medien, Transformationen und Konstruktionen, Berlin - New York, p. 292-312.

Brisson, L., 1996, Introduction à la philosophie du mythe. I. Sauver les mythes, Paris.

Cameron, A., 1995, Callimachus and his Critics, Princeton.

Caneva, S.G., 2007, Raccontare nel tempo. Narrazione epica e cronologia nelle Argonautiche di Apollonio Rodio, in S.G. Caneva, V. Tarenzi, Il lavoro sul mito nell'epica greca. Letture di Omero e Apollonio, Pisa, p. 67-135.

Carrière, J.C., 1969, Philadelphe ou Sôtêr? À propos d'un hymne de Callimaque, StudClas, 11, p. 85-93.

Chaniotis, A., 2003, The Divinity of Hellenistic Rulers, in A. Erskine (ed.), A Companion to the Hellenistic World, Malden - Oxford - Victoria, p. 431-446.

Chaniotis, A., 2007, La divinité mortelle d'Antiochos III à Téos, Kernos, 20, p. 153-171.

Clauss, J.J., 1986, Lies and Allusions: The Addressee and Date of Callimachus'Hymn to Zeus, CA, 5, p. 155-170.

Cole, T., 1967, Democritus and the Sources of Greek Anthropology, Atlanta (II ed. Oxford 1990).

Cuypers, M., 2004, Prince and Principle: The Philosophy of Callimachus' Hymn to Zeus, in M.A. Harder, R.F. Regtuit, G.C. Wakker (eds), Callimachus II, Leuven, p. 95-115.

D’Alessio, B., 1996, Callimaco, Milano.

Davies, J., 2002, The Interpretation of Hellenistic Sovereignties, in D. Ogden (ed.), The Hellenistic World: New Perspectives, London, p. 1-22.

Dillery, J., 1998, Hecataeus of Abdera: Hyperboreans, Egypt, and the "Interpretatio Graeca", Historia, 47.3, p. 255-275.

FinKelberG, M., 1998, The Birth of Literary Fiction in Ancient Greece, Oxford.

Floridi, L., 2004, Mendacità del mito e strategie encomiastiche nellInno a Zeus di Callimaco, in R. Pretagostini, E. Dettori (eds), La cultura ellenistica. L'opera letteraria e l'esegesi antica. Atti del convegno COFIN 2001, Università di Roma "Tor Vergata", 22-24 settembre 2003, Roma, p. 65-75.

Fraser, P.M., 1972, Ptolemaic Alexandria, Oxford.

Funrer, T., 1988, A Pindaric Feature in the Poems of Callimachus, AJPh, 109, p. 53-68.

Goldhill, S., 1986, Framing and Polyphony: Readings in Hellenistic Poetry, PCPS, 212, p. $25-52$.

Goldhill, S., 1991, The Poet's Voice: Essays on Poetics and Greek Literature, Cambridge. Навіснт, Ch., 19702, Gottmenschentum und Griechische Städte, München (I ed. 1956). Harder, M.A., 1992, Insubstantial Voices: Some Oservations on the Hymns of Callimachus, CQ, 42, p. 384-394. 
Harder, M.A., 2003, The Invention of Past, Present and Future in Callimachus'Aetia, Hermes, 131, p. 290-306.

Harder, M.A., 2010, Callimachus' Aetia, in J.J. Clauss, M. Cuypers (eds), A companion to Hellenistic literature, Malden MA, Oxford, p. 92-105.

Henrichs, A., 1984, The Sophists and Hellenistic Religion: Prodicus as the Spiritual Father of the Isis Aretalogies, $\mathrm{HsPh}, 88$, p. 139-158.

Herman, G., 1997, The Court Society of the Hellenistic Age, in P. Cartledge, P. Garnsey, E.S. Gruen (eds), Hellenistic Constructs: Essays in Culture, History, and Historiography, Berkeley - Los Angeles, p. 199-224.

Hopkinson, N., 1984, Callimachus'Hymn to Zeus, CQ, 34, p. 139-148.

Hose, M., 1997, Der alexandrinische Zeus. Zur Stellung der Dichtkunst im Reich der ersten Ptolemäer, Philologus, 141, p. 46-64.

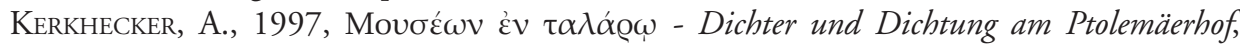
A\&A, 43, p. 124-144.

Kerkhecker, A., 1999, Callimachu' Book of Iambi, Oxford.

KокоLакIS, M., 1995, Zeus' tomb. An Object of Pride and Reproach, Kernos, 8, p. 126-130.

Lelli, E., 2004, Critica e polemiche letterarie nei Giambi di Callimaco, Alessandria.

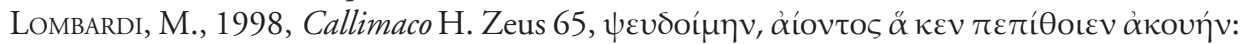
la poetica della verità e le menzogne credibili, RCCM, 40, p. 165-172.

Lüddecke, K.L.G., 1998, Contextualizing the Voice in Callimachus" "Hymn to Zeus», MD, 41, p. 9-33.

MA, J., 20022, Antiochos III and the Cities of Western Asia Minor, Oxford (I ed. 1999).

MA, J., 2003, Kings, in A. Erskine (ed.), A Companion to the Hellenistic World, Malden Oxford - Victoria, p. 177-195.

Mari, M., 2004, Il culto della personalità a Samo, tra Lisandro e Demetrio Poliorcete, in E. Cavallini (ed.), Samo: storia, letteratura, scienza. Atti delle giornate di studio, Ravenna, 14/16-11-2002, Ravenna, p. 177-196.

Mari, M., 2008 , The Ruler Cult in Macedonia, «Studi Ellenistici» 20, p. 219-268.

McLennan, G.R., Callimachus, Hymn to Zeus: Introduction and Commentary, Roma.

MeIllier, C., 1979, Callimaque et son temps. Recherches sur la carrière et la condition d'un écrivain à l'époque des premiers Lagides, Lille.

Mooren, L., 1983, The Nature of the Hellenistic Monarchy, in E. Van't Dack, P. Van Dessel, W. Van Gucht (eds), Egypt and the Hellenistic World. Proceedings of the International Colloquium, Leuven - 24-26 May 1982, Leuven, p. 205-240.

Morrison, A.D., The Narrator in Archaic Greek and Hellenistic Poetry, Cambridge.

MülLER, R.J., 1993, Überlegungen zur IEPA ANАГРАФН des Euhemeros von Messene, Hermes, 122.3, p. 276-300.

Müller, S., 2009, Das hellenistische Königspaar in der medialen Repräsentation Ptolemaios II und Arsinoe II, Berlin.

Murray, O., 1970, Hecataeus of Abdera and Pharaonic Kingship, JEA, 56, p. 141-171.

Murray, O., 1996, Hellenistic Royal Symposia, in P. Bilde, T. Engberg-Pedersen, L. Hannestad, J. Zahle (eds), Aspects of Hellenistic Kingship, Aarhus, p. 15-27.

Pellizer, E., 1993, La mitografia, in G. Cambiano, L. Canfora, D. Lanza (eds), Lo spazio letterario in Grecia antica, Vol. I.2, Roma, p. 283-303. 
PfeIfFer, S., 2008, Herrscher-und Dynastiekulte im Ptolemäerreich: Systematik und Einordnung der Kultformen, München.

PratT, L.H., Lying and Poetry from Homer to Pindar: Falsehood and Deception in Archaic Greek Poetics, Ann Arbor, MI.

Reinsch-Werner, H., 1976, Kallimachus Hesiodicus. Die Rezeption der hesiodischen Dichtung durch Kallimachus von Kyrene, Berlin.

Rice, E.E., 1983, The Grand Procession of Ptolemy Philadelphus, Oxford.

Richter, O., 1871, Kallimachu' Hymnen auf Zeus und Apollo: Zwei Momente im Leben des Ptolemaeus Philadelphus, Guben.

Rösler, W., 1980, Die Entdeckung der Fiktionalität in der Antike, Poetica, 12, p. 283-319.

Samuel, A.E., 1993, The Ptolemies and the Ideology of Kingship, in P. Green (ed.), Hellenistic History and Culture, Berkeley - Los Angeles, p. 168-210.

SpOerri, W., 1959, Späthellenistische Berichte über Welt, Kultur und Götter. Untersuchungen zu Diodor von Sizilien, Basel.

Stephens, S., 2003, Seeing Double. Intercultural Poetics in Ptolemaic Alexandria, Berkeley Los Angeles.

Strootman, R., 2010, The hellenistic Royal Court. Court Culture, Ceremonial and Ideology in Greece, Egypt and the Near East, 336-30 BCE, Diss Utrecht, http://igitur-archive.library. uu.nl/dissertations/2007-0725-201108/index.htm

Strootman, R., 2010, Literature and the Kings, in J.J. Clauss, M. Cuypers (eds), A Companion to Hellenistic Literature, Malden MA, Oxford, p. 30-45.

Thompson, D.J., 1973, Ptolemaic Oinochoai and Portraits in Faience: Aspects of the Ruler Cult, Oxford.

Vallauri, G., 1956, Evemero di Messene, Torino.

Van Nuffelen, P., Le culte royal de l'empire des Séleucides: une réinterprétation, Historia, 53.3, p. 278-301.

Vamvouri Ruffy, M., 2004, La fabrique du divin. Les Hymnes de Callimaque à la lumière des Hymnes homériques et des Hymnes épigraphiques, Liège.

Walbank, F. W., 1984, Monarchies and Monarchic Ideas, in F.W. Walbank, A.E. Astin (eds), The Cambridge Ancient History, VII.1, Cambridge, p. 62-100.

Weber, G., 1992, Poesie und Poeten an den Höfen vorhellenisticher Monarchen, Klio, 74, p. 25-77.

Weber, G., 1993, Dichtung und höfische Gesellfschaft: Die Rezeption von Zeitgeschichte am Hof der ersten drei Ptolemäer, Stuttgart.

Weber, G., 1995, Herrscher, Hof und Dichter: Aspekte der Legitimierung und Repräsentation hellenisctischer Könige am Beispiel der ersten drei Antigoniden, Historia, 44, p. 283-316.

Weber, G., 1997, Interaktion, Repräsentation und Herrschaft: Der Königshof im Hellenismus, in A. Winterling (ed.), Zwischen "Haus" und "Staat": Antike Höfe im Vergleich, München, p. 27-71.

Wilamowitz-Moellendorf, U. von, 1924, Hellenistische Dichtung in der Zeit des Kallimachos, Berlin.

WiniarczyK, M., 1991, Euhemeri Messenii Reliquiae, Stuttgart - Leipzig.

WiniarczyK, M., 2002, Euhemerus. Leben, Werk und Nachwirkung, München - Leipzig. 